Adv. Studies Theor. Phys., Vol. 7, 2013, no. 3, 131 - 134

HIKARI Ltd, www.m-hikari.com

\title{
The Force Law Design of Artificial Physics Optimization for Starting Population Selection for GSAT
}

\author{
Anna Gorbenko \\ Department of Intelligent Systems and Robotics \\ Ural Federal University \\ 620083 Ekaterinburg, Russia \\ gorbenko.ann@gmail.com \\ Vladimir Popov \\ Department of Intelligent Systems and Robotics \\ Ural Federal University \\ 620083 Ekaterinburg, Russia \\ Vladimir.Popov@usu.ru
}

\begin{abstract}
GSAT is a well-known satisfiability search algorithm which uses some random functions. In this paper, we consider an artificial physics optimization for computing a function of starting population.
\end{abstract}

PACS: 07.05.Mh

Keywords: artificial physics optimization, GSAT, satisfiability

Investigation of efficient satisfiability algorithms and encoding different hard problems as instances of SAT has caused considerable interest (see e.g. [1] - [16]). GSAT is a well-known satisfiability algorithm [17]. The GSAT algorithm is a search method for finding a truth assignment for the variables of CNF. GSAT uses some random functions. One of such functions is a function of starting population of truth assignments. In this paper, we consider an intelligent algorithm for computing a function of starting population of truth assignments. This algorithm is based on artificial physics optimization.

Note that artificial physics optimization algorithm (see [18]) consists of three procedures: 
- initialization;

- calculation force;

- motion.

A swarm of individuals are sampled randomly from a problem space in the initialization. Masses of individuals should be calculated in the procedure of calculation force. The procedure motion uses the total force to calculate individual velocity. Since a felicitous design of force law can drive individuals search problem space intelligently and efficiently, the main advantage of the artificial physics optimization consists in the proper design of force law.

We consider force law in the general polynomial form

$$
\begin{gathered}
F(x[1], \ldots, x[n], m[1], \ldots, m[n])= \\
\sum_{i=1}^{s} \alpha[a[i, 1,1], \ldots, a[i, n, n], b[i, 1], \ldots, b[i, n]] \\
\prod_{1 \leq j \leq n, 1 \leq k \leq n, j \neq k}(|x[j]-x[k]|)^{a[i, j, k]} \prod_{p=1}^{n} m[p]^{b[i, p]}
\end{gathered}
$$

where $x[i]$ is defined as the position of individual $i, m[i]$ is defined as the mass of individual $i$, and $\alpha, a, b$ are constants.

Let

$$
f(z[1], \ldots, z[t])
$$

be a CNF. We assume that $n>t$, for any possible value of $t$. Let \#occ ${ }^{+}(f, z[i])$ be the number of positive occurrences of $z[i]$ in $f, \# o c c^{-}(f, z[i])$ be the number of occurrences of $\neg z[i]$ in $f$. For any $f$, we assume that

$$
m[i]=\left\{\begin{array}{l}
\sum_{j=1}^{t} \beta[j]\left(\# o c c^{+}(f, z[i]), \# o c c^{-}(f, z[i])\right) t^{j}, 1 \leq i \leq t \\
0, i>t .
\end{array}\right.
$$

We use genetic algorithms to determine the values of the parameters $\alpha, \beta, a$, $b$. In particular, we use the following algorithms for design of force law.

1. A genetic algorithm (GA[1]) to determine the values of

$$
\beta[1], \ldots, \beta[t] .
$$

2. A genetic algorithm (GA[2]) to determine the values of

$$
[a[i, 1,1], \ldots, a[i, n, n], b[i, 1], \ldots, b[i, n]] .
$$

3. A genetic algorithm (GA[3]) to determine the values of

$$
\alpha[a[i, 1,1], \ldots, a[i, n, n], b[i, 1], \ldots, b[i, n]] .
$$

As values of

$$
z[1], z[2], \ldots, z[t]
$$


we consider $\{0,1\}$-discretized values of

$$
x[1], x[2], \ldots, x[t] .
$$

In our experiments, we consider standard GSAT and GSAT with the artificial physics optimization for starting population of truth assignments (GSATAPO). For GSAT and GSAT-APO we consider the same number of truth assignments and the same set of CNF. Also, we use the same number of inversions. Selected experimental results are given in Table 1.

\begin{tabular}{|l|l|lllll|}
\hline & & $G[1]=10^{4}$ & $G[1]=10^{5}$ & $G[1]=10^{5}$ & $G[1]=10^{6}$ & $G[1]=10^{6}$ \\
& GSAT & $G[2]=10^{4}$ & $G[1]=10^{5}$ & $G[1]=10^{6}$ & $G[1]=10^{5}$ & $G[1]=10^{6}$ \\
& & $G[3]=10^{4}$ & $G[1]=10^{5}$ & $G[1]=10^{6}$ & $G[1]=10^{5}$ & $G[1]=10^{6}$ \\
\hline$N$ & $76 \%$ & $77 \%$ & $84 \%$ & $85 \%$ & $94 \%$ & $95 \%$ \\
\hline
\end{tabular}

Table 1: Experimental results for GSAT and GSAT-APO with different numbers of generations of genetic algorithms, where $N$ is a number of solved CNFs, $G[i]$ is a number of generations of genetic algorithm $\mathrm{GA}[i]$.

ACKNOWLEDGEMENTS. The work was partially supported by Analytical Departmental Program "Developing the scientific potential of high school" 8.1616.2011.

\section{References}

[1] A. Gorbenko and V. Popov, Footstep Planning for Humanoid Robots, Applied Mathematical Sciences, 6 (2012), 6567-6571.

[2] A. Gorbenko, M. Mornev, V. Popov, and A. Sheka, The problem of sensor placement for triangulation-based localisation, International Journal of Automation and Control, 5 (2011) 245-253.

[3] A. Gorbenko and V. Popov, SAT Solvers for the Problem of Sensor Placement, Advanced Studies in Theoretical Physics, 6 (2012) 1235-1238.

[4] A. Gorbenko and V. Popov, Clustering Algorithm in Mobile Ad Hoc Networks, Advanced Studies in Theoretical Physics, 6 (2012) 1239-1242.

[5] A. Gorbenko, M. Mornev, and V. Popov, Planning a Typical Working Day for Indoor Service Robots, IAENG International Journal of Computer Science, 38 (2011) 176-182.

[6] A. Gorbenko and V. Popov, The Problem of Selection of a Minimal Set of Visual Landmarks, Applied Mathematical Sciences, 6 (2012) 4729-4732. 
[7] A. Gorbenko and V. Popov, On the Problem of Placement of Visual Landmarks, Applied Mathematical Sciences, 6 (2012) 689-696.

[8] A. Gorbenko and V. Popov, On the Longest Common Subsequence Problem, Applied Mathematical Sciences, 6 (2012), 5781-5787.

[9] A. Gorbenko and V. Popov, On the Problem of Sensor Placement, Advanced Studies in Theoretical Physics, 6 (2012) 1117-1120.

[10] A. Gorbenko and V. Popov, The Longest Common Parameterized Subsequence Problem, Applied Mathematical Sciences, 6 (2012), 2851-2855.

[11] A. Gorbenko and V. Popov, The set of parameterized k-covers problem, Theoretical Computer Science, 423 (2012), 19-24.

[12] A. Gorbenko and V. Popov, The Far From Most String Problem, Applied Mathematical Sciences, 6 (2012), 6719-6724.

[13] A. Gorbenko and V. Popov, Multi-agent Path Planning, Applied Mathematical Sciences, 6 (2012), 6733-6737.

[14] A. Gorbenko and V. Popov, Programming for Modular Reconfigurable Robots, Programming and Computer Software, 38 (2012) 13-23.

[15] A. Gorbenko, V. Popov, and A. Sheka, Localization on Discrete Grid Graphs, Lecture Notes in Electrical Engineering, 107 (2012), 971-978.

[16] A. Gorbenko and V. Popov, Task-resource Scheduling Problem, International Journal of Automation and Computing, 9 (2012), 429-441.

[17] B. Selman, H. Levesque, and D. Mitchell, A new method for solving hard satisfiability problems, Proceedings of the Tenth National Conference on Artificial Intelligence, (1992), 440-446.

[18] L. Xie and J. Zeng, The Performance Analysis of Artificial Physics Optimization Algorithm Driven by Different Virtual Forces, ICIC Express Letters, 4 (2010), 239-244.

\section{Received: December 3, 2012}

